\title{
Effectiveness of Optic Decompression by Endoscopic Endonasal Approach on Patients with Traumatic Fracture of Optic Canal
}

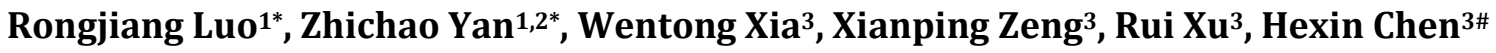 \\ ${ }^{1}$ Department of Ophthalmology, The First Affiliated Hospital, Sun Yat-sen University, Guangzhou, China \\ ${ }^{2}$ Department of Ophthalmology, The Second Affiliated Hospital, Guangzhou Medical University, Guangzhou, China \\ ${ }^{3}$ Institute of Otorhinolaryngology, Sun Yat-sen University, Guangzhou, China \\ Email: "chenhexin9999@126.com
}

How to cite this paper: Luo, R.J., Yan, Z.C., Xia, W.T., Zeng, X.P., Xu, R. and Chen, H.X. (2017) Effectiveness of Optic Decompression by Endoscopic Endonasal Approach on Patients with Traumatic Fracture of Optic Canal. Open Access Library Journal, 4: e3682.

https://doi.org/10.4236/oalib.1103682

Received: May 19, 2017

Accepted: June 7, 2017

Published: June 10, 2017

Copyright $\odot 2017$ by authors and Open Access Library Inc.

This work is licensed under the Creative Commons Attribution International License (CC BY 4.0).

http://creativecommons.org/licenses/by/4.0/

\begin{abstract}
Traumatic fracture of optic canal is a severe injury to the optic nerve and often causes permanent visual loss, and it is difficult to treat. This study is to evaluate the effectiveness of decompression of optic canal by nasal endoscope. Operation of optic canal decompression was performed on 210 cases (212 sides) with traumatic fracture of optic canal. Clinical effectiveness was assessed based on improvement of visual acuity. After following up for 6 months to 2 years, visual improvement with more than one visual acuity level was observed on 101 sides (47.6\%). Among them, an excellent effect (increasing 2 levels of visual acuity) was achieved on 72 sides (34.0\%). Among 133 sides performed within 5 days of injury, 61 sides (45.9\%) showed excellent effect (improved 2 levels of visual acuity). In contrast, when operation was performed after 5 days of injury, only 11 out of 79 sides (13.9\%) showed excellent effect with improvement of 2 levels of visual acuity. And the overall effectiveness between the operations were performed within and after 5 days of injury was different significantly $(P<0.05)$. Therefore, endonasal endoscopic optic decompression for traumatic optic neuropathy is an effective measure for patients with traumatic optical canal fracture. Operation within 5 days of injury significantly increases the success rate.
\end{abstract}

\section{Subject Areas \\ Ophthalmology, Otorhinolaryngology}

\section{Keywords}

Nasal Endoscope, Optic Nerve Decompression, Optic Nerve Injury 


\section{Introduction}

Traumatic optic neuropathy (TON) is an impact injury to the optic nerve and often a devastating cause of permanent visual loss after blunt or penetrating injury [1] [2] [3]. Traumatic fracture of optic canal is a severe type of TON. The choice of treatment for traumatic fracture of optic canal is controversial [4] [5] [6]. The treatment of the condition includes observation, the use of corticosteroids and surgical decompression of the optic nerve [1] [6] [7] [8] [9]. The surgery to reduce the pressure in the canal involves the craniotomy either from the medial wall of the orbit or from the nasal cavity via endoscope surgery [10]. The endoscopic endonasal approach has the advantage of short surgical path, clear view of local tissues, and less damage [11]. It has been considered as an effective clinical practice [5] [12] [13] [14]. Whereas the anatomy surrounding of the fractures of optic canal is complicated, operation requires sophisticated skills. Importantly, the impact of the timing of operation on the success of surgery is less clear. Our department has treated 210 patients (212 sides) from October 2003 to March 2010. Here we reported that endoscopic optic decompression via endonasal approach for traumatic fracture of optic canal is effective. Early treatment significantly increases the success rate of the operation.

\section{Materials and Methods}

\subsection{Patients}

Between October 2003 and March 2010, 210 patients (212 sides) with optic canal fracture were enrolled in our department. The inclusion criteria included clinical findings of visual loss in patients due to head or mid-facial trauma or the presence of an ipsilateral afferent pupillary defect with normal eyesight in the other eye. Patients were excluded if their posttraumatic visual loss was not related to optic nervedys function, such as an open globe, traumatic cataract, vitreous hemorrhage, retinal detachment, choroidal rupture, or orbital compartment syndrome. Among the patients, the youngest patient is 4.5 years old, while the oldest patient is 64 years old. There were 164 male and 46 female patients. The imaging examinations confirmed the fracture of optic canal and intact eyeball in coronal and horizontal position by computed tomography (CT) scan. Among these patients, 158 cases (160 sides) were complicated with orbital wall fracture, 35 cases $(22.2 \%)$ had zygomatic arch fracture, 41 cases (25.9\%) had intracranial hematoma, and 6 patients (3.8\%) suffered from cerebrospinal fluid rhinorrhea concurrently. These patients were hospitalized after injury for 2 hours to 22 days. Among 210 patients, 133 sides were operated within 5 days of injury. The study protocol followed the principles in the Declaration of Helsinki. Informed consent was obtained from the patient prior to all treatments and investigations.

\subsection{Surgery}

Surgical operation for optic decompression by endoscopic endonasal approach was carried out between 3 hours and 3 days after admission. For tracheal intuba- 
tion and general anesthesia, the operators opened the ethmoid sinus to expose sphenoid sinus and cleared hemorrhage and effusion in sphenoid sinus. The optic canal in sphenoid sinus was located and the existence of Onodi cells was determined. When the position of the optic canal cannot be identified, sphenoid sinus mucosa near the orbital apex is peeled. The uplift of optic nerve bone tube would then appear (Figure 1). If the optic canal position were still unclear, the back board of the medial orbit wall was removed to the orbital apex (Figure 2), according the position and move towards of orbital apex to locate the position of optic canal. At last bone tube was attenuated by steel drill. The rest thin bone tube was gently dissected to open the optic nerve sheath membrane. Then the total tendon ring was cut.

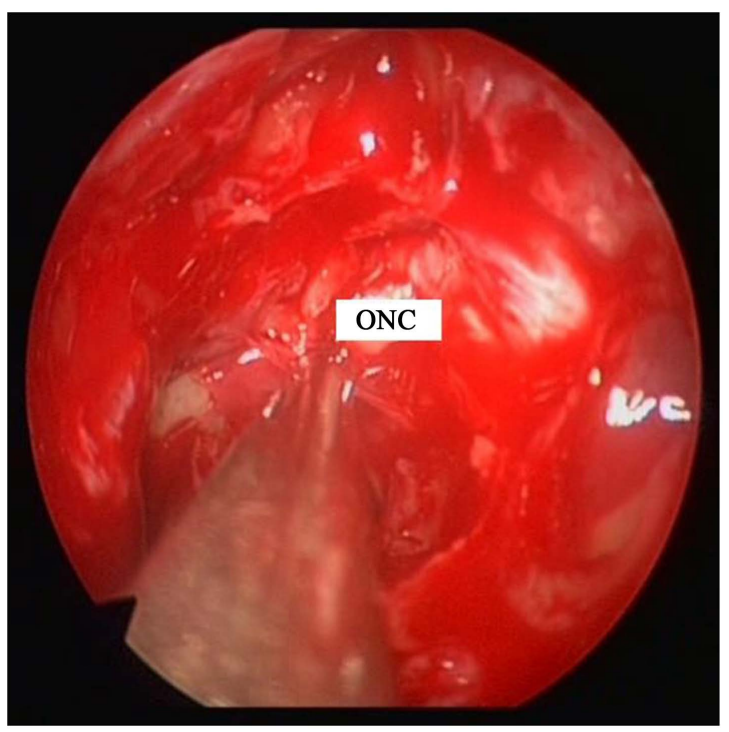

Figure 1. Demonstration of optical nerve canal, after mucosa of lateral wall in sphenoidal sinus were avulsed. ONC: optical nerve canal.

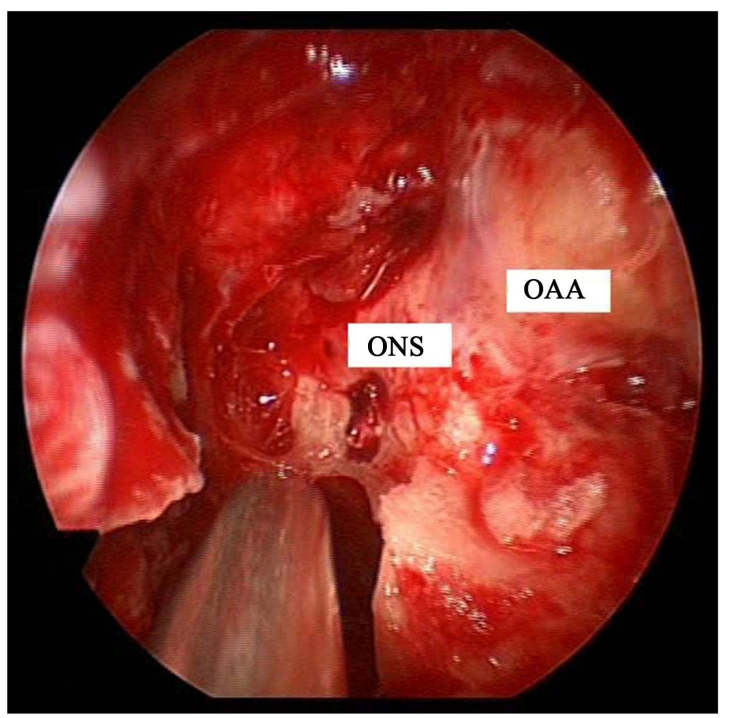

Figure 2. Exposure of orbital apex aponeurosis and optic nerve sheaths. OAA: orbital apex aponeurosis; ONS: optic nerve sheaths. 


\subsection{Medications before and after Surgery}

Before the operation, patients were given methylprednisolone (10 to $15 \mathrm{mg} / \mathrm{kg} / \mathrm{day}$ ) for one time. After operation, patients were given half dose of methylprednisolone in the first three days. These patients also received gastric mucosa protectants at same time.

\subsection{The Assessment of Vision}

Visual acuity (VA) levels include blindness, light perception (LP), hand motions (HM), finger counting (FC) and every rising line in visible vision chart by using LogMAR letters. Promoting a level and the improvement of the vision are regarded to be effective. These cases included 77 cases of blinds (36.3\%), 43 cases of LP (20.3\%), 37 cases of HM (17.5\%), 42 cases of FC (19.8\%), and 13 cases of visible vision chart (6.1\%). There were 37 patients (17.5\%) with vision defect.

\subsection{Statistical Analysis}

Therapeutic efficacy was considered to be a dependent variable. The patients were divided into two groups according to the time of the operation ( $\leq 5$ days vs. $>5$ days). The two group comparisons were performed, the basic demographic data of the two groups, and both adjusted and unadjusted for baseline visual acuity. All statistical analyses were performed using SPSS 13.0. $P$ values $<$ 0.05 were considered to be statistically significant.

\section{Results}

There were 101 sides (47.6\%) with visual improvement of more than one visual acuity level were observed in this study, and among them there were 72 sides (34.0\%) with visual improvement of more than two visual acuity level. According to different timing of operation on the success rate of surgery, 61 sides (45.9\%) improved 2 levels of visual acuity were observed, and 12 sides (9.0\%) improved 1 level of visual acuity were achieved when the operation was performed within 5 days of injury. And the overall effectiveness was seen in 73 sides (54.9\%). In contrast, when the operation was performed later than 5 days after injury, 11 out of 79 sides (13.9\%) showed improvement of 2 levels of visual acuity, and there were 17 out of 79 sides (21.5\%) which showed one level improvement of visual acuity, and the overall effectiveness was seen in 28 out of 79 sides (35.4\%). The overall effectiveness between the operations performed within and after 5 days of injury were different significantly $(P<0.05)$. There was no any severe surgical complication occurred in all patients.

\section{Discussion}

In this report, we analyzed the outcome of 212 sides of optical canal decompression on 210 patients. The overall effectiveness was observed on 101 sides following optical canal operation with visual improvement of more than one visual acuity level (47.6\%). Importantly, among them there were 72 sides which achieved an excellence effect by increasing 2 levels of visual acuity (34.0\%). This 
demonstrates the effectiveness of optical canal decompression via endonasal endoscopic approach.

We further analyzed the impact of the timing of operation on the success rate of surgery. Among 210 patients (212 sides), 133 sides were operated within 5 days of injury. Excellent effect as determined by improving 2 levels of visual acuity was achieved on 61 sides (45.9\%). Good effect as determined by improving 1 level of visual acuity was achieved on 12 sides (9.0\%). The overall effectiveness was seen in 73 sides (54.9\%). In contrast, when the operation was performed later than 5 days after injury, the success rate decreased significantly. There were 79 out of 212 sides which were operated after 5 days (between 5 to 14 days) of injury. In this population, 11 out of 79 sides (13.9\%) showed excellent effect with improvement of 2 levels of visual acuity. There were 17 out of 79 sides (21.5\%) which showed good effects with one level improvement of visual acuity. The overall effectiveness was seen in 28 out of 79 sides $(35.4 \%)(P<0.05$ in comparison to who has operation performed within 5 days of injury in the population). The molecular mechanisms underlying such drastic different outcome in these two populations remain to be determined. Earlier animal studies in rats revealed that after intraorbital optic nerve transection, retinal ganglion cells survive for 5 days and then die abruptly in large numbers, largely due to apoptosis [15]. It is likely that human optical nerve functions are similar to that seen in rats. The damage of optical nerve in the initial 5 days of injury is reversible. When the time exceeds 5 days, a cascade of molecular events such as apoptosis occurs which leads to the irreversible nature of the damage. Our finding highlights the importance of early intervention following optical injury.

In our practice of optic canal decompression by nasal endoscope, we felt that several skills are helpful in minimizing surgical complications. The foremost important issue is the identification of optic canal in external wall of sphenoid sinus. This often is the bottleneck for the beginner surgeons. There are three steps in clinical practice that are important for identifying the optic canal. Step one is the careful examination of CT image before surgery. Before operation patients should receive sinus and orbital apex coronal CT in order to identify the location of Onodi cells. The coronal image is helpful in understanding the morphological characteristics of Onodi cells [16] [17]. Proper identification could avoid blind searching for the optic nerve in sphenoid sinus which is a common cause of damage in cavernous sinus including damaging of the internal carotid artery. Step two is to tear off the sphenoid sinus mucosa. Owing to the variation of sphenoid sinus and the mucosal edema and hemorrhage in the sphenoid sinus, sometimes the exposure of optic canal is not clear. Striping sphenoid sinus mucosa away along lateral sphenoid sinus could fully expose the bone. Then the outline of optic canal will be exposed (Figure 1). Step three is to remove the back board of the medial orbit wall. If the optic nerve bone tube could not be identified after step 1 and 2, lamina papyracea which locates at the segment a posterius of orbit medial wall should be removed. Orbital apex along the orbital fascia should be identified. Then the bone of optic uplift can be removed. Based 
on the direction of orbital fascia and the bone mark in sphenoid sinus, an accurate identification of optic nerve bone tube could be made (Figure 2).

After making an accurate judgment of the location of the optic canal, the position of the fracture line should be checked. Gentle and accurate management of the optic nerve bone are essential in preventing further optic nerve injury by the fractures. Similarly, using low temperature saline wash in the application of steel drill can prevent the production of heat. Overexertion of the fracture line should be avoided to prevent nerve injury by fracture segmenta. When bone is thin enough, it can be stripped off by elevator. When the haemorrhage occurred in the optic nerve bone and surrounding structures, a gentle suppression of cotton piece with physiological saline is useful. The adrenaline cotton piece should be avoided because it can cause eye artery spasm leading to further vision damage. In the event of no obvious optic canal fracture was observed, the incision of both tendon ring and nerve sheath membrane is necessary as recommended by Thaker A [18]. Incision of tendon ring and nerve sheath membrane at the same time would achieve a better clinical effect.

Cavernous sinus is below optic canal [19]. When optic canal bone fracture line linked with the bone below, bone needs to be stripped. However, cautions should be applied to avoid the haemorrhage of cavernous sinus (Figure 3). The bleeding should be tackled using saline cotton pieces. The upper part of the optic canal is next to the skull base. If the fracture line linked with the skull base bone, stripping the optic nerve bone tube is required. Caution should be applied to avoid the injury of meninx and prevent the cerebrospinal fluid rhinorrhoea. In the event of cerebrospinal fluid rhinorrhoea, middle turbinate mucous membrane could be repaired by using biological glue stick. Considering that the optic nerve is often bare, a gelatin sponge is often used to gently prevent the oppression of the optic nerve. Eye artery enters into optic canal and intoendocranium. In these locations there are inherent nutrition artery and optic nerve nutrition

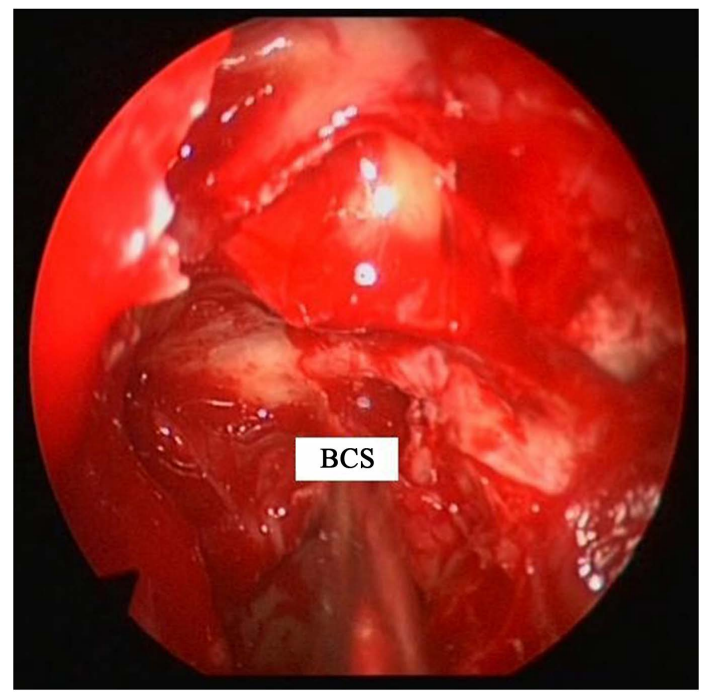

Figure 3. Bleeding in cavernous sinus of optic canal inferior wall during operation. BCS: bleeding for cavernous sinus. 
branch of sheath artery. These arteries are often located in the optic ventral side or outside of optical nerve. Accordingly, when the dura mater needs to be cut, the best parts of incision are above the lateral wall of optic nerve sheath. This could avoid damaging the eye arteries [20].

In summary, endonasal endoscopic optic decompression for TON is an effective measure for patients with traumatic optical canal fracture, and operation within 5 days of injury significantly increases the success rate.

\section{Acknowledgements}

We thank Dr. Huanquan Liao (the First Affiliated Hospital, Sun Yat-sen University) for manuscript review and discussions.

\section{References}

[1] Kumaran, A.M., Sundar, G. and Chye, L.T. (2015) Traumatic Optic Neuropathy: A Review. Craniomaxillofacial Trauma \& Reconstruction, 8, 31-41. https://doi.org/10.1055/s-0034-1393734

[2] Singman, E.L., Daphalapurkar, N., White, H., Nguyen, T.D., Panghat, L., Chang, J., et al. (2016) Indirect Traumatic Optic Neuropathy. Military Medical Research, 3, 2. https://doi.org/10.1186/s40779-016-0069-2

[3] Yang, Q.T., Zhang, G.H., Liu, X., Ye, J. and Li, Y. (2012) The Therapeutic Efficacy of Endoscopic Optic Nerve Decompression and Its Effects on the Prognoses of 96 Cases of Traumatic Optic Neuropathy. The Journal of Trauma and Acute Care Surgery, 72, 1350-1355. https://doi.org/10.1097/TA.0b013e3182493c70

[4] Chaon, B.C. and Lee, M.S. (2015) Is There Treatment for Traumatic Optic Neuropathy? Current Opinion in Ophthalmology, 26, 445-449. https://doi.org/10.1097/ICU.0000000000000198

[5] Chen, F., Zuo, K., Feng, S., Guo, J., Fan, Y., Shi, J., et al. (2014) A Modified Surgical Procedure for Endoscopic Optic Nerve Decompression for the Treatment of Traumatic Optic Neuropathy. North American Journal of Medical Sciences, 6, 270-273.

[6] Yu-Wai-Man, P. (2015) Traumatic Optic Neuropathy-Clinical Features and Management Issues. Taiwan Journal of Ophthalmology, 5, 3-8. https://doi.org/10.1016/j.tjo.2015.01.003

[7] Levin, L.A., Beck, R.W., Joseph, M.P., Seiff, S. and Kraker, R. (1999) The Treatment of Traumatic Optic Neuropathy: The International Optic Nerve Trauma Study. Ophthalmology, 106, 1268-1277. https://doi.org/10.1016/s0161-6420(99)00707-1

[8] Warner, N. and Eggenberger, E. (2010) Traumatic Optic Neuropathy: A Review of the Current Literature. Current Opinion in Ophthalmology, 21, 459-462. https://doi.org/10.1097/ICU.0b013e32833f00c9

[9] Vaitheeswaran, K., Kaur, P. and Garg, S. (2014) Minimal Invasive Transcaruncular Optic canal Decompression for Traumatic Optic Neuropathy. Orbit, 33, 456-458. https://doi.org/10.3109/01676830.2014.950282

[10] Sarkies, N. (2004) Traumatic Optic Neuropathy. Eye, 18, 1122-1125. https://doi.org/10.1038/sj.eye.6701571

[11] Horiguchi, K., Murai, H., Hasegawa, Y., Mine, S., Yamakami, I. and Saeki, N. (2010) Endoscopic Endonasal Trans-Sphenoidal Optic Nerve Decompression for Traumatic Optic Neuropathy-Technical Note. Neurologia Medico-Chirurgica, 50, 518522. https://doi.org/10.2176/nmc.50.518 
[12] Zhang, Q., Lu, H., Li, G., Gong, S., Wang, Y., Meng, W., et al. (2015) Long-Term Efficacy of Nasal Endoscopic Optic Nerve Decompression for Traumatic Optic Neuropathy. Journal of Clinical Otorhinolaryngology, Head, and Neck Surgery, 29, 1082-1085.

[13] Peng, A., Li, Y., Hu, P. and Wang, Q. (2011) Endoscopic Optic Nerve Decompression for Traumatic Optic Neuropathy in Children. International Journal of Pediatric Otorhinolaryngology, 75, 992-998.

[14] Kong, D.S., Shin, H.J., Kim, H.Y., Chung, S.K., Nam, D.H., Lee, J.I., et al. (2011) Endoscopic Optic Canal Decompression for Compressive Optic Neuropathy. Journal of Clinical Neuroscience: Official Journal of the Neurosurgical Society of Australasia, 18, 1541-1545.

[15] Berkelaar, M., Clarke, D.B., Wang, Y.C., Bray, G.M. and Aguayo, A.J. (1994) Axotomy Results in Delayed Death and Apoptosis of Retinal Ganglion Cells in Adult Rats. The Journal of Neuroscience: The Official Journal of the Society for Neuroscience, 14, 4368-4374.

[16] Hart, C.K., Theodosopoulos, P.V. and Zimmer, L.A. (2009) Anatomy of the Optic Canal: A Computed Tomography Study of Endoscopic Nerve Decompression. The Annals of Otology, Rhinology, and Laryngology, 118, 839-844. https://doi.org/10.1177/000348940911801203

[17] Unal, B., Bademci, G., Bilgili, Y.K., Batay, F. and Avci, E. (2006) Risky Anatomic Variations of Sphenoid Sinus for Surgery. Surgical and Radiologic Anatomy, 28, 195-201. https://doi.org/10.1007/s00276-005-0073-9

[18] Thaker, A., Tandon, D.A. and Mahapatra, A.K. (2009) Surgery for Optic Nerve Injury: Should Nerve Sheath Incision Supplement Osseous Decompression? Skull Base: Official Journal of North American Skull Base Society, 19, 263-271. https://doi.org/10.1055/s-0028-1114299

[19] Isolan, G.R., Krayenbuhl, N., de Oliveira, E. and Al-Mefty, O. (2007) Microsurgical Anatomy of the Cavernous Sinus: Measurements of the Triangles in and around It. Skull Base: Official Journal of North American Skull Base Society, 17, 357-367. https://doi.org/10.1055/s-2007-985194

[20] Sandu, K., Monnier, P. and Pasche, P. (2012) Anatomical Landmarks for Transnasal Endoscopic Skull Base Surgery. European Archives of Otorhinolaryngology: Official Journal of the European Federation of Otorhinolaryngology Societies, 269, 171-178. https://doi.org/10.1007/s00405-011-1698-4

\section{Open Access Library}

Submit or recommend next manuscript to OALib Journal and we will provide best service for you:

- Publication frequency: Monthly

- 9 subject areas of science, technology and medicine

- Fair and rigorous peer-review system

- Fast publication process

- Article promotion in various social networking sites (LinkedIn, Facebook, Twitter, etc.)

- Maximum dissemination of your research work

Submit Your Paper Online: Click Here to Submit

Or Contact service@oalib.com 